\title{
Divisibility Criteria for Class Numbers of Imaginary Quadratic Fields Whose Discriminant Has Only Two Prime Factors
}

\author{
A. Pekin \\ Department of Mathematics, Faculty of Science, Istanbul University, 34134 Istanbul, Turkey \\ Correspondence should be addressed to A. Pekin, aypekin@istanbul.edu.tr
}

Received 17 October 2012; Accepted 4 November 2012

Academic Editor: Haydar Akca

Copyright @ 2012 A. Pekin. This is an open access article distributed under the Creative Commons Attribution License, which permits unrestricted use, distribution, and reproduction in any medium, provided the original work is properly cited.

We will prove a theorem providing sufficient condition for the divisibility of class numbers of certain imaginary quadratic fields by $2 g$, where $g>1$ is an integer and the discriminant of such fields has only two prime divisors.

\section{Introduction}

Let $K=Q(\sqrt{D})$ be the quadratic fields with discriminant $D$ and $h=h(D)$ its class number. In the narrow sense, the class number of $K$ is denoted by $h^{+}(D)$, where, if $D>0$, then $h^{+}(D)=$ $2 h(D)$ and the fundamental unit $\varepsilon_{D}$ has norm 1, otherwise $h^{+}(D)=h(D)$. If the discriminant of $|D|$ has two distinct prime divisors, then by the genus theory of Gauss the 2-class group of $K$ is cyclic. The problem of the divisibility of class numbers for number fields has been studied by many authors. There are Hartung [1], Honda [2], Murty [3], Nagel [4], Soundararajan [5], Weinberger [6], Yamamoto [7], among them. Ankeny and Chowla [8] proved that there exists infinitely many imaginary quadratic fields each with class numbers divisible by $g$ where $g$ is any given rational integer. Later, Belabas and Fouvry [9] proved that there are infinitely many primes $p$ such that the class number of the real quadratic field $K=Q(\sqrt{p})$ is not divisible by 3. Furthermore, many authors $[7,10-13]$ have studied the conditions for $h^{+}(D)$ to be divisible by $2^{n}$ when the 2-class group of $K$ is cyclic. However the criterion for $h^{+}(D)$ to be divisible by $2^{n}$ is known for only $n \leq 4$ and the existence of quadratic fields with arbitrarily large cyclic 2-class groups is not known yet. Recently, Byeon and Lee [14] proved that there are infinitely many imaginary quadratic fields whose ideal class group has an element of order $2 g$ and whose discriminant has only two prime divisors. In this paper, we will prove a theorem that the order of the ideal class group of certain imaginary quadratic field is divisible by 
$2 g$. Moreover, we notice that the discriminant of these fields has only different two prime divisors. Finally, we will give a table as an application to our main theorem.

\section{Main Theorem}

Our main theorem is the following.

Theorem 2.1. Let $D=p q$ be square-free integer with primes $p \equiv q \equiv 1(\bmod 4)$. If there is a prime $r \equiv 1(\bmod 8)$ satisfying $(D / r)=1$, then $t \mid h(D)$ for at least positive integer $t$ where $t \geq 2$. theorems.

In order to prove this theorem we need the following fundamental lemma and some

Lemma 2.2. If $D$ is of the form $p \cdot q$ where $p$ and $q$ are primes $p \equiv q \equiv 1(\bmod 4)$, then there is $a$ prime $r \equiv 1(\bmod 8)$ such that $(D / r)=1$.

Proof. Let $a$ and $b$ be quadratic nonresidues for $p$ and $q$ are primes such that $(a / p)=-1$, $(b / q)=-1$, where () denotes Legendre symbol and $\mathrm{g} \cdot \mathrm{c} \cdot \mathrm{d}(p, q)=1$. Therefore, by Chinese Remainder Theorem, we can write $w \equiv a(\bmod p), w \equiv b(\bmod q)$ for a positive integer $w$. Now, we consider the numbers of the form $p q k+w$ such that $p q k_{0}+w \equiv 1(\bmod 8)$ for some $1 \leq k_{0} \leq 8$. Since $p q k_{0}+w$ are distinct residues $\bmod (8)$ for some $1 \leq k_{0} \leq 8$, then we get $p q\left(8 n+k_{0}\right)+w=8 p q n+p q k_{0}+w, n \geq 0$. We assert that $\mathrm{g} \cdot \mathrm{c} \cdot \mathrm{d}\left(8 p q, p q k_{0}+w\right)=1$. Really, we suppose that $\mathrm{g} \cdot \mathrm{c} \cdot \mathrm{d}\left(8 p q, p q k_{0}+w\right)=m>1$, then there is a prime $s$ such that $s \mid m$, and so we have $s|8 p q, s| p q k_{0}+w$. Thereby this follows that $s=2, p$ or $q$. But since $p q k_{0}+w \equiv 1(\bmod 8)$, then $s \neq 2$ and $s \mid m$; this is in contradiction with $w \equiv a(\bmod p), w \equiv$ $b(\bmod q)$. Therefore, $\mathrm{g} \cdot \mathrm{c} \cdot \mathrm{d}\left(8 p q, p q k_{0}+w\right)=1$ holds. Thus, by the Dirichlet theorem on primes, there is a prime $r$ satisfying $r=p q\left(8 n+k_{0}\right)+w=8 p q n+p q k_{0}+w$. Hence, it is seen that $r \equiv 1(\bmod 8)$.

The following theorem is generalized by Cowles [15].

Theorem 2.3. Let $r, m$, $t$ be positive integers with $m>1$ and $t>1$, and let $n=r^{2}-4 m^{t}$ be squarefree and negative. If $m^{c}$ is not the norm of a primitive element of $O_{K}$ whenever c properly divides $t$, then $t \mid h(n)$.

Cowles proved this theorem by using the decomposition of the prime divisors in $O_{K}$. But Mollin has emphasized in [16] that it contains some misprints and then he has provided the following theorem which is more useful in practise than Theorem 2.4.

Theorem 2.4. Let $n$ be a square-free integer of the form $n=r^{2}-4 m^{t}$ where $r, m$, and $t$ are positive integers such that $m>1$ and $t>1$. If $r^{2} \leq 4 m^{t-1}(m-1)$, then $t \mid h(n)$.

Theorem 2.5. Let $n$ be a square-free integer, and let $m>1, t>1$ be integers such that

(i) $\mp m^{t}$ is the norm of a primitive element from $K=Q(\sqrt{n})$,

(ii) $\mp m^{c}$ is not the norm of a primitive element from $K$ for all c properly dividing $t$,

(iii) if $t=|m|_{2}$, then $n \equiv 1(\bmod 8)$.

Then $t$ divides the exponent of $\psi_{K}$, where $\psi_{K}$ is the class group of $K$.

\section{Proof of Main Theorem}

Now we will provide a proof for the fundamental theorem which is more practical than all of the works above mentioned. 
Table 1

\begin{tabular}{lcccc}
\hline$D$ & $p$ & $q$ & $r$ & $h(D)$ \\
\hline 65 & 5 & 13 & 17 & 8 \\
1165 & 5 & 233 & 41 & 20 \\
3341 & 13 & 257 & 41 & 72 \\
10685 & 5 & 2137 & 73 & 116 \\
30769 & 29 & 1061 & 41 & 112 \\
45349 & 101 & 449 & 17 & 168 \\
95509 & 149 & 641 & 17 & 176 \\
97309 & 73 & 1333 & 89 & 216 \\
102689 & 29 & 3541 & 73 & 496 \\
125009 & 41 & 3049 & 17 & 504 \\
18497 & 53 & 349 & 41 & 168 \\
20453 & 113 & 181 & 17 & 116 \\
223721 & 137 & 1633 & 97 & 496 \\
378905 & 5 & 75781 & 41 & 592 \\
567137 & 17 & 333613 & 89 & 640 \\
650117 & 13 & 50009 & 17 & 848 \\
735929 & 373 & 1973 & 41 & 1664 \\
847085 & 5 & 169417 & 73 & 936 \\
874589 & 241 & 3629 & 17 & 1160 \\
875705 & 5 & 175141 & 41 & 1328 \\
876461 & 53 & 16537 & 73 & 1584 \\
971081 & 109 & 8909 & 17 & 1464 \\
971413 & 29 & 33497 & 73 & 336 \\
978809 & 13 & 75293 & 89 & 1728 \\
987169 & 97 & 10177 & 17 & 624 \\
999997 & 757 & 1321 & 17 & 380 \\
\hline & & & &
\end{tabular}

Proof. From the assumption of Lemma 2.2, it follows that there is suitable prime $r$ with $r \equiv$ $1(\bmod 8)$ such that $(D / r)=1$. However, from the properties of the Legendre symbol, we can write $\left(D y^{2} / r^{2}\right)=1$ for any integer $y$. Since $(2, r)=1$, then we have $\left(D y^{2} / r^{t}\right)=1$. Therefore, there are integers $x=a / 2, y=b / 2$ such that the equation $x^{2}-D y^{2}=\mp r^{t}$ has a solution in integers. Hence, we can write $a^{2}-D b^{2}=\mp 4 r^{t}$, where $a \equiv b(\bmod 2)$. From this equation, it is seen that $r^{t}$ is the norm of a primitive element of $O_{K}$, and, then by Theorem 2.5, $t$ divides $h(n)$.

We have the following results.

Corollary 3.1. Let $D$ be a square-free and negative integer in the form of $D=n^{2}-4 r^{2 g}=p \cdot q$ with $n>1, g>1$ are positive integers and $p, q, r$ are primes such that $p \equiv q \equiv 1(\bmod 4), r \equiv 1(\bmod 8)$. If $r^{2 g}$ is the norm of a primitive element of $O_{K}$, then the order of the ideal class group of $K=Q(\sqrt{D})$ is $2 g$.

Corollary 3.2. Let $D$ be a square-free and negative integer in the form of $D=p \cdot q$, then there exists exactly 34433 imaginary quadratic fields satisfying assertion of the main theorem.

\section{Table}

The above-mentioned imaginary quadratic fields $K=Q(\sqrt{D})$ correspond to some values of $D\left(5 \leq D \leq 10^{6}\right)$ which are given in Table 1 . We have provided a table of the examples 
to illustrate the results above, using $C$ programming language. Moreover, it is easily seen that the class numbers of imaginary quadratic fields of $K=(Q \sqrt{D})$ are divisible by $2 g$ from Table 1.

\section{Acknowledgment}

This work was partially supported by the scientific research project with the number IUYADOP 12368.

\section{References}

[1] P. Hartung, "Proof of the existence of infinitely many imaginary quadratic fields whose class number is not divisible by $n$," Journal of Number Theory, vol. 6, pp. 276-278, 1974.

[2] T. Honda, "A few remarks on class numbers of imaginary quadratic number fields," Osaka Journal of Mathematics, vol. 12, pp. 19-21, 1975.

[3] M. R. Murty, "The ABC conjecture and exponents of class groups of quadratic fields," in Number Theory, vol. 210 of Contemporary Mathematics, pp. 85-95, American Mathematical Society, Providence, RI, USA, 1998.

[4] T. Nagel, "Über die Klassenzahl imaginär quadratischer Zahlkörper," Abhandlungen aus dem Mathematischen Seminar der Universität Hamburg, vol. 1, pp. 140-150, 1992.

[5] K. Soundararajan, "Divisibility of class numbers of imaginary quadratic fields," Journal of the London Mathematical Society. Second Series, vol. 61, no. 3, pp. 681-690, 2000.

[6] P. J. Weinberger, "Real quadratic fields with class numbers divisible by n," Journal of Number Theory, vol. 5, pp. 237-241, 1973.

[7] Y. Yamamoto, "Divisibility by 16 of class number of quadratic fields whose 2-class groups are cyclic," Osaka Journal of Mathematics, vol. 21, no. 1, pp. 1-22, 1984.

[8] N. C. Ankeny and S. Chowla, "On the divisibility of the class number of quadratic fields," Pacific Journal of Mathematics, vol. 5, pp. 321-324, 1955.

[9] K. Belabas and E. Fouvry, "Sur le 3-rang des corps quadratiques de discriminant premier ou presque premier," Duke Mathematical Journal, vol. 98, no. 2, pp. 217-268, 1999.

[10] P. Barrucand and H. Cohn, "Note on primes of type $x^{2}+32 y^{2}$, class number, and residuacity," Journal für die Reine und Angewandte Mathematik, vol. 238, pp. 67-70, 1969.

[11] H. Bauer, "Zur berechnung der 2-klassenzahl der quadratischen Zahlkörper mit genau zwei verschiedenen diskriminantenprimteilern," Journal für die Reine und Angewandte Mathematik, vol. 248, pp. 42-46, 1971.

[12] H. Hasse, "Über die teilbarkeit durch $2^{3}$ der Klassenzahl imaginärquadratischer Zahlkörper mit genau zwei verschiedenen diskriminantenprimteilern," Journal für die Reine und Angewandte Mathematik, vol. 241, pp. 1-6, 1970.

[13] P. Kaplan, K. S. Williams, and K. Hardy, “Divisibilité par 16 du nombre des classes au sens strict des corps quadratiques réels dont le deux-groupe des classes est cyclique," Osaka Journal of Mathematics, vol. 23, no. 2, pp. 479-489, 1986.

[14] D. Byeon and S. Lee, "Divisibility of class numbers of imaginary quadratic fields whose discriminant has only two prime factors," Japan Academy. Proceedings. Series A. Mathematical Sciences, vol. 84, no. 1, pp. 8-10, 2008.

[15] M. J. Cowles, “On the divisibility of the class number of imaginary quadratic fields," Journal of Number Theory, vol. 12, no. 1, pp. 113-115, 1980.

[16] R. A. Mollin, "Diophantine equations and class numbers," Journal of Number Theory, vol. 24, no. 1, pp. 7-19, 1986. 


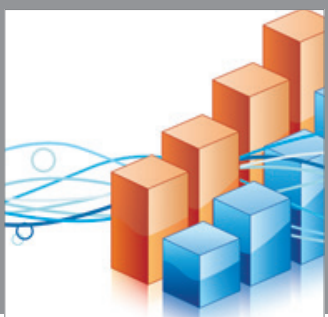

Advances in

Operations Research

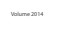

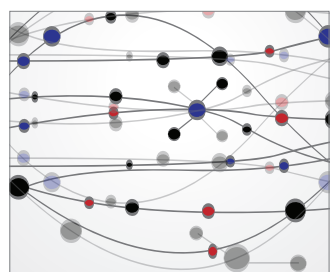

\section{The Scientific} World Journal
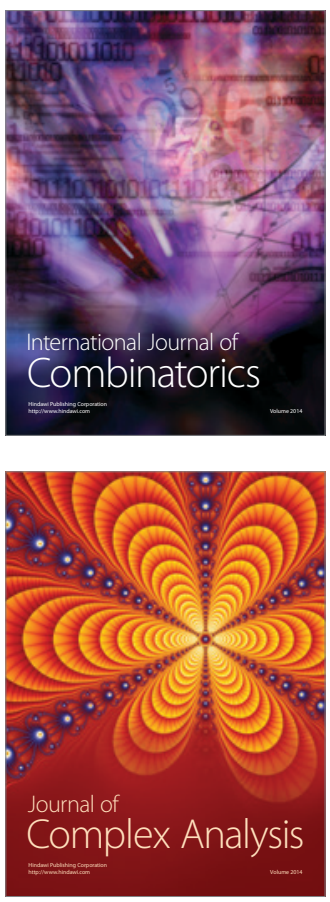

International Journal of

Mathematics and

Mathematical

Sciences
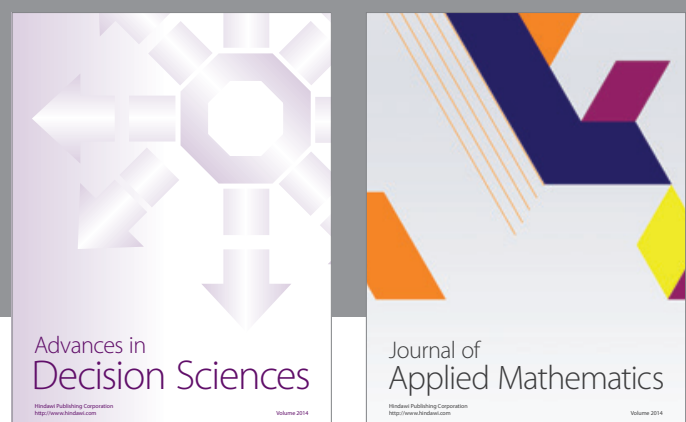

Journal of

Applied Mathematics
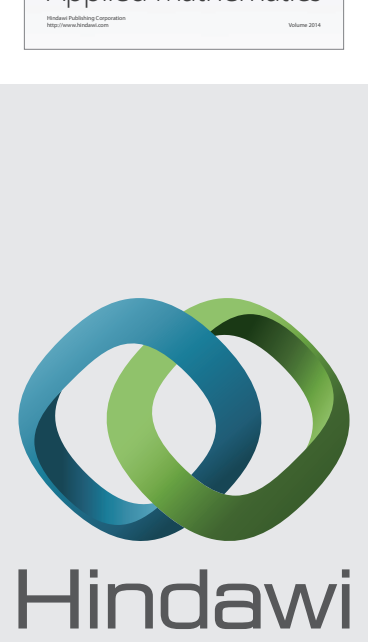

Submit your manuscripts at http://www.hindawi.com
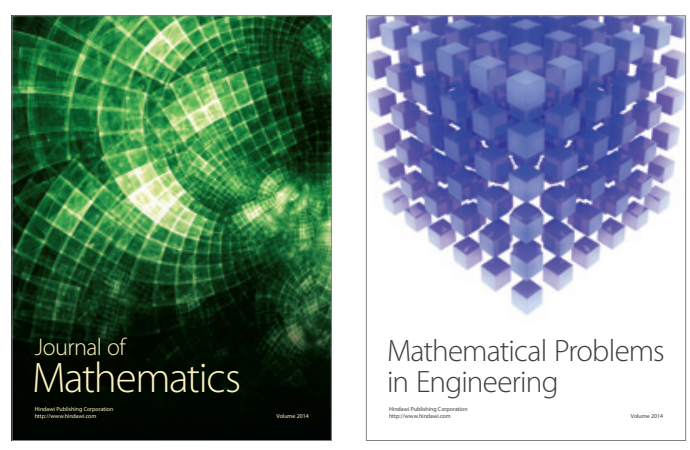

Mathematical Problems in Engineering
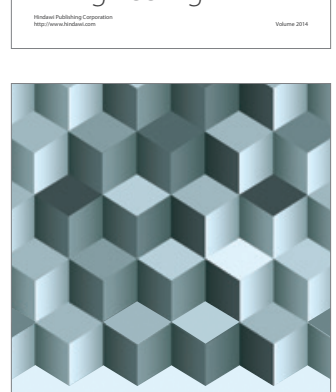

Journal of

Function Spaces
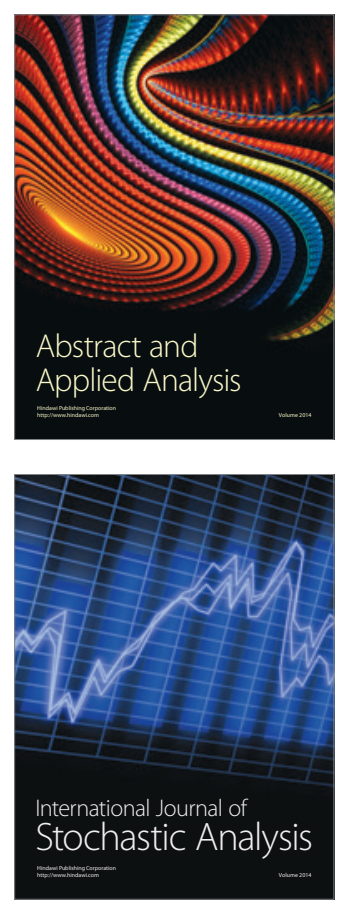

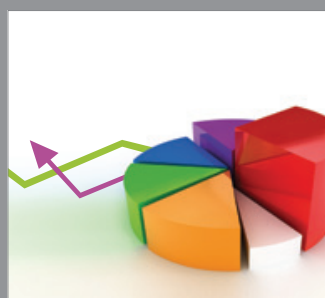

ournal of

Probability and Statistics

Promensencen
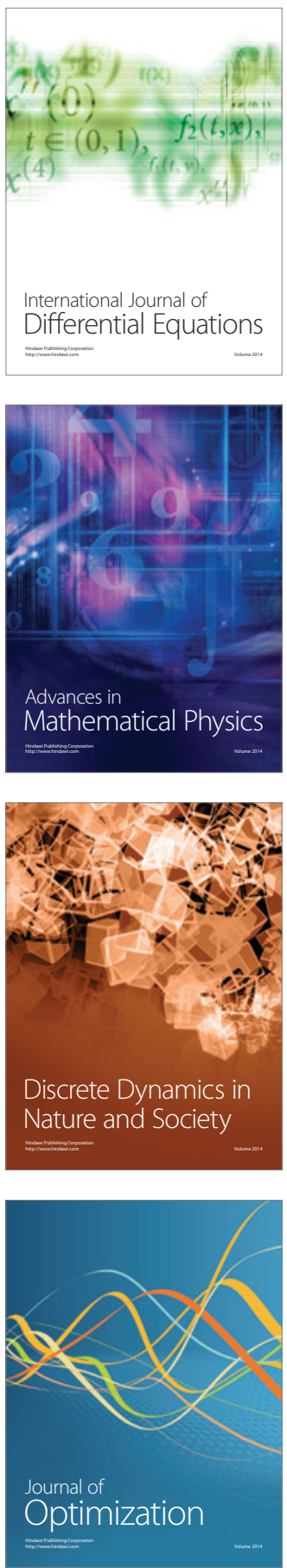